\title{
Childhood cancer and factors related to prolonged diagnostic intervals: a Danish population-based study
}

\author{
J M Ahrensberg ${ }^{*, 1,2,3}$, F Olesen ${ }^{1}$, R P Hansen ${ }^{1,3}$, H Schrøder $^{4}$ and P Vedsted ${ }^{1,3}$ \\ ${ }^{1}$ Research Unit for General Practice, Aarhus University, Bartholins Allé 2, 8000 Aarhus, Denmark; ${ }^{2}$ Section for General Practice, \\ Department of Public Health, Aarhus University, Bartholins Allé 2, 8000 Aarhus, Denmark; ${ }^{3}$ Research Centre for Cancer Diagnosis \\ in Primary Care, Aarhus University, Bartholins Allé 2, 8000 Aarhus C, Denmark and ${ }^{4}$ Department of Paediatrics, Aarhus University \\ Hospital, Brendstrupvej 100, 8200 Aarhus N, Denmark
}

Background: Early diagnosis of childhood cancer provides hope for better prognoses. Shorter diagnostic intervals (DI) in primary care require better knowledge of the association between presenting symptoms, interpretation of symptoms and the wording of the referral letter.

Methods: A Danish nationwide population-based study. Data on 550 children aged $<15$ years with an incident cancer diagnosis (January 2007-December 2010) were collected through questionnaires to parents (response rate $=69 \%$ ) and general practitioners (GPs) (response rate $=87 \%$ ). The DI from the first presentation in general practice until diagnosis was categorised as short or long based on quartiles. Associations between variables and long Dls were assessed using logistic regression.

Results: The GPs interpreted symptoms as 'vague' in $25.4 \%$, 'serious' in $50.0 \%$ and 'alarm' in $19.0 \%$ of cases. Symptom interpretation varied by cancer type $(P<0.001)$ and was associated with the $\mathrm{DI}(P<0.001)$. Vomiting was associated with a shorter DI for central nervous system (CNS) tumours, and pain with a longer DI for leukaemia. Referral letter wording was associated with DI $(P<0.001)$; the shortest Dls were observed when cancer suspicion was raised in the letter.

Conclusion: The GPs play an important role in recognising early signs of childhood cancer as their symptom interpretation and referral wording have a profound impact on the diagnostic process.

Cancer is one of the principal causes of child death in developed countries (Kaatsch, 2010). To achieve the best prognosis, a seamless pathway facilitating early diagnosis is essential as diagnosis delay may have adverse effects on, for example, the immune system, causing an increased risk of cancer therapy side effects (Goyal et al, 2004). Even if a delayed diagnosis may have little influence on the prognosis, patient and parental anxiety is relieved by a rapid diagnosis (Dixon-Woods et al, 2001; Goyal et al, 2004).

However, a fundamental problem in early diagnosis is that symptoms tend to be vague and may resemble frequently seen symptoms of benign conditions (Punt, 2004; Raab and Gartner,
2009). Furthermore, the positive predictive values (PPVs) of the presenting symptoms are extremely low (Dommett et al, 2012). Vague and misinterpreted symptoms may therefore lead to delay from the first presentation to diagnosis, that is, increase the length of the diagnostic interval (DI).

The visitation in paediatric departments and the clinical diagnosing may depend upon several factors, for example, the diagnostic capacity of the unit in question. In 2007-2010, when this study was performed, the paediatric visitation at hospitals was based on how the general practitioner (GP) described the symptoms and the possible urgency, and it was the paediatric consultant who decided how to prioritise the referral of the 
child. In late 2008, fast-track pathways were introduced in Denmark, implying that the GP could refer to a fast-track pathway in case of cancer suspicion. Thus, the GP's interpretation and description of the clinical picture and actual wording of the referrals, for example, the choice of words like 'alarm' or 'serious disease', could speed up the diagnostic process.

The aim of this study was to investigate the association between the DI and presenting symptoms, symptom interpretation and referral wording for children with a pathway involving general practice to test if long DIs are more likely when symptoms are vague and referral wording does not indicate cancer suspicion.

\section{MATERIALS AND METHODS}

Overall study design. The study was conducted as a populationbased cohort study. Patients were sampled from the Danish Childhood Cancer Registry (DCCR). Data were obtained from registries and questionnaires. We used the unique civil registration number (CRN) to link registers at the individual level. The DI was defined as the time from the first presentation to general practice to the date of diagnosis (Weller et al, 2012).

Study participants. Information on children aged $0-14$ years registered with an incident cancer in the DCCR from 1 January 2007 to 31 December 2010 was obtained. Included in the study were children with malignant cancers and benign tumours of the central nervous system (CNS) (included diagnoses according to the Danish version of the International Classification of Diseases (ICD-10) (World Health Organisation, 2011): C00.0-C96.9 (malignant), and D32, D33, D35.2-4, D42, D43 and D44.3-5 (benign tumours of the CNS)). A total of 29 children were excluded (Figure 1) on the basis of the following criteria: diagnosis established within the first week of life, incorrect CRN, living in an institution or outside Denmark; recurrent cancer; severe comorbidity before diagnosis (congenital hypothyreosis, Wilms' tumour aniridia syndrome, von Hippel-Lindau syndrome, neurofibromatosis and pulmonary fibrosis). Children were also excluded if the GP advised against sending the questionnaire to the parents.

Register data. The DCCR is a national register in Denmark. All Danish departments of paediatric oncology report to the DCCR.
The DCCR holds information on ICD-10 codes and the date of diagnosis for all children with malignancies and benign tumours of the CNS. The date of the diagnosis is defined as the date of bone marrow aspirate (leukaemia) or diagnostic imaging (solid tumour). We linked the cohort with the Danish Civil Registration System (Pedersen et al, 2006), which holds information on residence and vital status of all residents in Denmark, and with the Danish National Health Service Register (Olivarius et al, 1997), which provided information on the names and addresses of the GPs with whom the children were listed at the time of diagnosis.

Questionnaire data. The child's GP was informed about the study before data collection. A questionnaire sent to the parents contained items on parental education and an item requesting consent to sending a questionnaire to the GP (Q (GP)). The Q (GP) asked if the GP had been totally or partly involved in the process of establishing the child's cancer diagnosis (yes/no) and requested a detailed description of the patient's first symptom presentation, clinical findings and specific dates, for example, date of first presentation, referral and other milestones in the diagnostic process. Apart from symptoms and clinical findings, the Q (GP) also focused on the GP's 'clinical intuition' that 'something was wrong'. Furthermore, the Q (GP) included questions on the GP's symptom interpretation, that is, whether the symptoms according to his or her clinical judgement were 'alarm symptoms, that is, indicating cancer disease'; 'serious symptoms, that is, indicating severe disease, but not cancer' or 'vague symptoms, that is, not indicating severe disease'. Finally, the Q (GP) included questions on the wording of the referral letter ('cancer suspicion'; 'serious illness, no cancer suspicion' or 'something wrong').

The literature search revealed no questionnaires that were suitable for this study. The questionnaires were designed by the research group on the basis of literature studies, preceding interviews, clinical experience and studies on time intervals in the diagnosis of cancer in adults (these were performed at the Research Unit for General Practice, Aarhus University). Both questionnaires were pilot-tested two times (among researchers at the Department and on a sample of GPs) and were adjusted accordingly (link to questionnaires can be seen at the end of the paper).

Data were collected in the following order: First, the parent questionnaire was sent. Updated information on the child's vital status (alive/deceased) was collected on the day before sending the

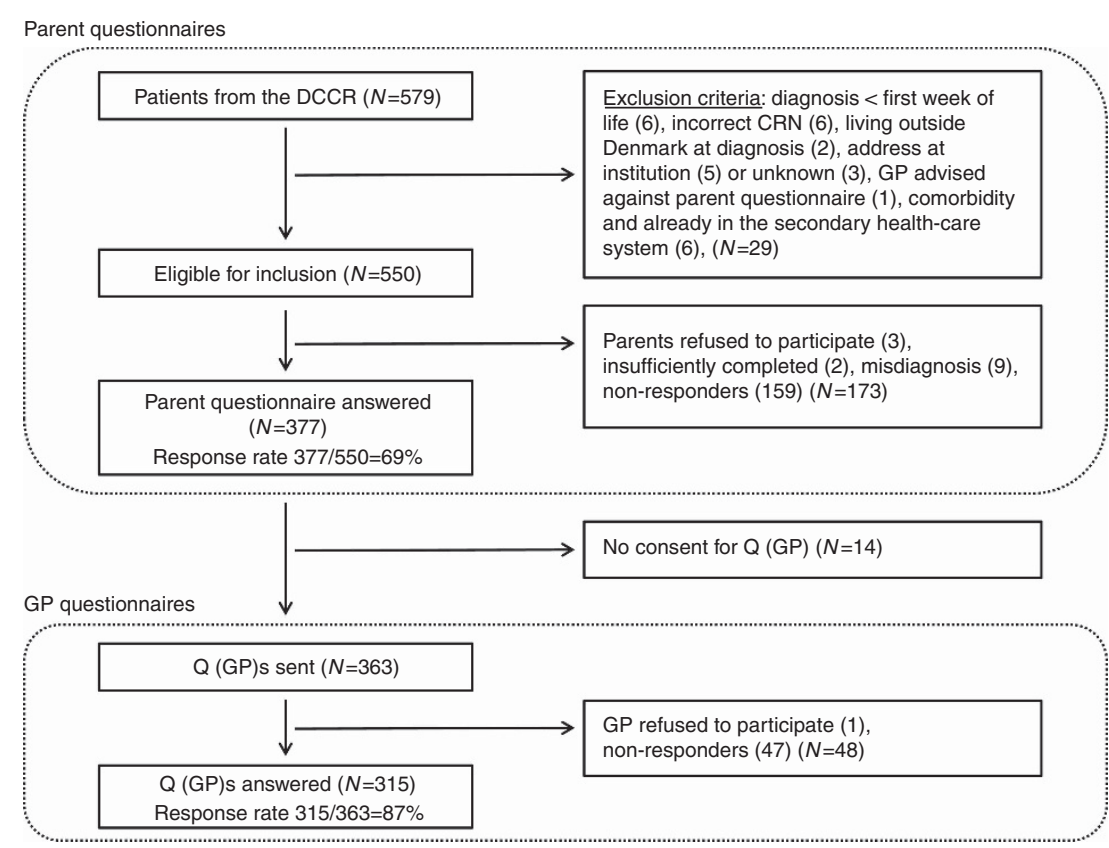

Figure 1. Flowchart. 
questionnaire. Questionnaire data on children diagnosed in 2007 and 2008 were collected in December 2009. The remaining data were collected consecutively, but at least 3 months after the child had been diagnosed. If the child had died, a specific cover letter and a questionnaire for bereaved parents were used. In such cases, the questionnaire was sent at least 6 months after parental bereavement.

The Q (GP) was sent if parents gave consent (96\%; 363 of 377 ). The Q (GP) was sent to the person who was the child's GP on the date of diagnosis. In practices with more than one GP, the GP most familiar with the child was asked to complete the Q (GP). The data collection ended in September 2011. Non-responding parents and GPs received a reminder after 3 weeks. The GPs received a $£ 28$ (240 DKK) fee for their participation. A flowchart of the questionnaire part of the study is presented in Figure 1.

Statistical analyses. Analyses of the DI were restricted to pathways involving a GP. The date of the first presentation to general practice was missing for a single patient with a CNS tumour. The DI was measured in days (median, interquartile interval (IQI)). Differences between groups were tested using the Kruskal-Wallis test. The grouping variables included gender, age, parental education (the mother's education was used as a proxy for socioeconomic position; Krieger et al, 1997), cancer type, calendar year, presenting symptoms, clinical findings, symptom interpretation and referral wording. The educational level was categorised as low (no or few vocational courses), medium (vocational training or education of up to 3 years) or high (higher education of 3 years or more). Categorical associations were tested using $\chi^{2}$ tests.

The association between general and nonspecific symptoms and the DI was estimated for the total group of childhood cancer patients. The association between cancer-related symptoms and the DI was estimated for the following specific cancers: leukaemia, lymphoma and CNS tumours. Regression analyses were used to calculate the association between long DIs and the independent variables. Long DIs were defined as the interval found in the fourth quartile. We used robust variance estimates to adjust the models for gender, age, diagnostic group and patient clustering according to GP. Prevalence ratios (PRs) with $95 \%$ confidence intervals $(95 \%$ CIs) were preferred to odds ratios, which may overestimate the associations because the prevalence of the outcome measure was above 20\% (Barros and Hirakata, 2003). We used generalised linear models with log link with the Bernoulli family, that is, we modelled the PR. A $P$-value of 0.05 or less was defined as statistically significant. Data were analysed using Stata 11.2 (Stata Software, version 11.2, StataCorp, College Station, TX, USA).

Ethics. The study was approved by the Danish Data Protection Agency (J.no. 2008-41-2956) and by the Committee of Multipractice Studies that recommended GPs to participate in the study. This project did not need approval from the Danish research ethics committee system. The National Board of Health (today: the Danish Health and Medicines Authority) did not support direct data collection from the GPs without parental consent, implying that parents had to respond to a questionnaire before data could be collected from general practice.

\section{RESULTS}

Descriptive data. A total of 550 children with incident cancers were eligible for inclusion (Figure 1). The parents of 377 (68.5\%) children diagnosed with cancer filled in a questionnaire and 363 allowed us to send a Q (GP). The Q (GP) was returned for 315 children (response rate $86.8 \%$ ) (Figure 1). The GPs were involved in the diagnostic process in 253 children. When the questionnaires were sent out, $242(95.7 \%)$ of the children were alive. The composition of the study population is shown in Table 1 .
Analyses revealed that non-responding mothers had a lower mean age $(P=0.036)$ than mothers in the responding group. Apart from this, no statistically significant difference was observed between responding and non-responding parents in terms of gender, age, vital status, calendar year, time since diagnosis, father's age and diagnostic groups (data not shown).

Symptoms were interpreted as either 'serious' (i.e. severe disease was indicated in 50.0\%), as 'alarm' (i.e. cancer disease was indicated in $19.0 \%$ ) or as 'vague' (i.e. severe disease was not indicated in $25.4 \%$ of cases); the item was missing for 14 patients (corresponding to 5.6\%). Symptom interpretation varied significantly by diagnostic group $(P<0.001)$ (Table 2$)$.

Alarm symptoms were more commonly reported for lymphoma patients $(43.2 \%)$ than for bone tumour patients $(5.3 \%)$, for whom vague symptoms were most often reported (47.4\%). Cancer suspicion was raised in the referral letter for 44 (36.7\%) of the patients with haematological cancer, for $8(15.7 \%)$ of the patients with a CNS tumour and for none of the patients with a bone tumour (data not shown).

\section{DIs for all cancer types}

Patient- and cancer-related factors. The median DI varied statistically significantly between age groups; the highest likelihood of a prolonged DI was observed in children aged 5-9 years (Table 1). Moreover, the DI varied by cancer type. The shortest DI was seen in children with leukaemia and the longest in children with bone and CNS tumours. Gender and socioeconomic status were not associated with DI length.

The DI length tended to decrease between 2007-2008 and 2009-2010, especially regarding the 75th percentile in which the DI was reduced from 60 to 42 days (Table 1 ).

Association between presented symptoms, GP interpretation and wording of referral letter. The DI was statistically significantly shorter when fatigue was reported than when fatigue was not reported (Table 2). Children presenting with pain tended to experience longer DIs than when pain was not present. No single symptom was identified as a statistically significant predictor of a long DI.

Symptom interpretation was highly indicative of DI length (Table 2), and the likelihood of a long DI was 3.0 times higher when GPs categorised presented symptoms as 'vague' rather than 'alarm' symptoms. Furthermore, the wording of the referral letter was strongly associated with the DI (Table 2); the median DI was nearly a month when the wording 'something wrong' was used, but only a week when 'suspicion of cancer' was stated.

DIs for individual cancer types. For children with leukaemia, the presence of fatigue, anaemia or bruising was associated with a shorter DI than when no such symptoms or clinical findings were reported (Table 3). The likelihood of a long DI was statistically significantly higher in the presence of pain than when pain was not reported. It should be noted that the median DIs for leukaemia patients with or without pain were similar. For children with lymphomas, however, the presence of pain, as opposed to no pain, was associated with a statistically significantly shorter DI. For children with CNS tumours, the presence of vomiting was statistically significantly associated with a shorter DI than when no such symptom was reported.

DISCUSSION

Main findings. The GP's symptom interpretation and the wording of the referral letter were strongly associated with DI length. The presence of fatigue was associated with a shorter DI, whereas no specific symptoms were associated with a prolonged 


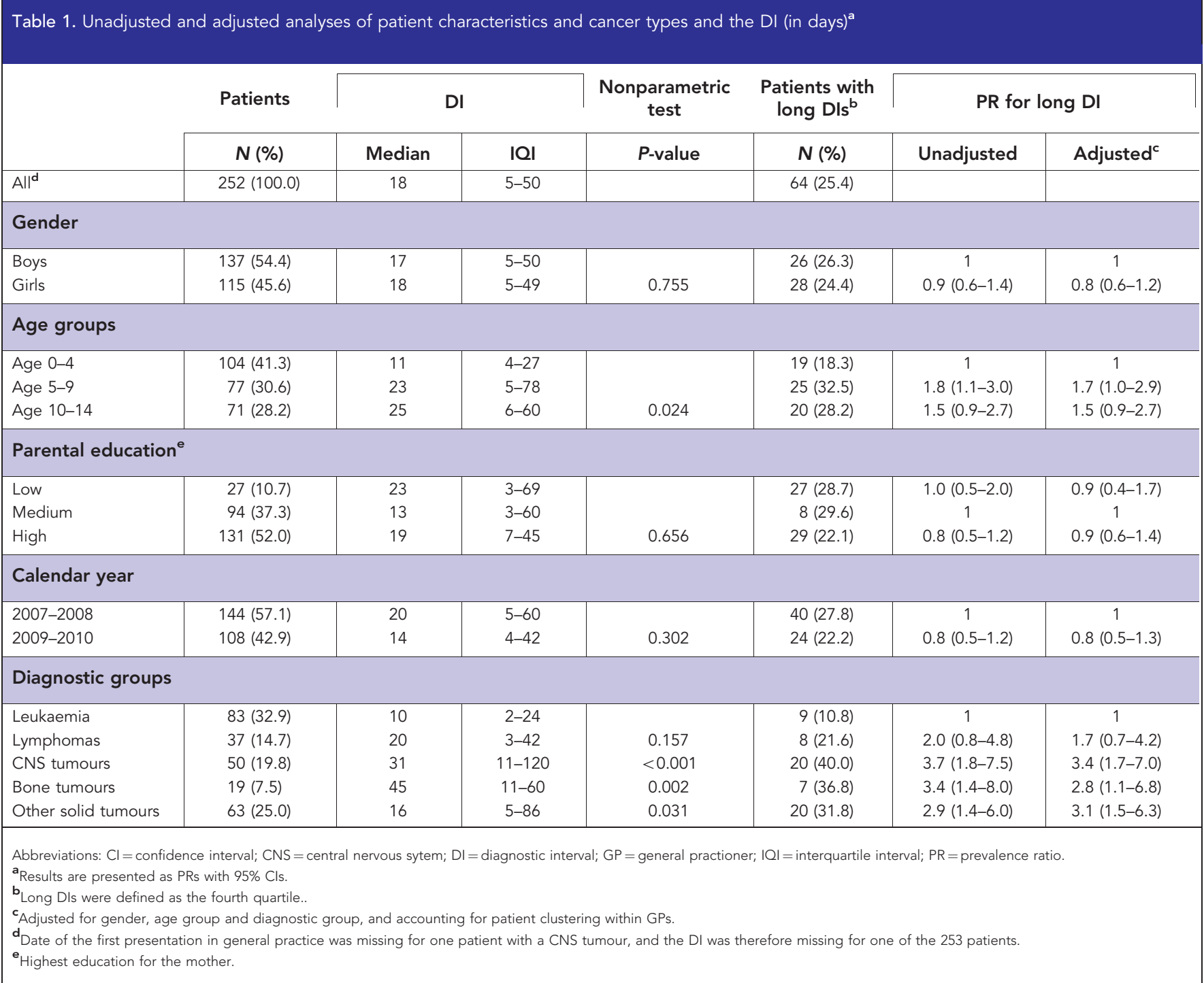

DI for the total group of children with cancer. However, children with leukaemia were more likely to experience a long DI when pain was reported than when pain was not reported. Paediatrics are aware that bone pains are common in leukaemia patients. In primary care, however, other explanations for (bone) pain are much more plausible, for example, minor traumas, and the PPV of 'pain' therefore tends to be very low. The shortest DIs among children with brain tumours were observed when vomiting was present, whereas rather long DIs were reported in children without this symptom. This could imply that the GP - and the secondary health-care system - may have been misled in the interpretation of the clinical picture.

Despite the formerly reported low predictive values of presented symptoms of childhood cancer (Dommett et al, 2012), GPs nevertheless interpreted presented symptoms as 'serious' symptoms (i.e. indication of severe disease in $50 \%$ of cases) or 'alarm' symptoms (i.e. indication of cancer disease in $19 \%$ of cases). This indicates that GP symptom interpretation depends on both the specific symptoms prompting the encounter and other factors, such as the medical history, the clinical picture and the way parents express their concern.

Strengths and weaknesses. This study included children with an incident cancer diagnosis recruited from a whole nation over a period of 4 years. Children with benign CNS tumours were included because their presenting symptoms and the way these children are managed in primary care are most likely independent of histology. This approach allowed us to include a relatively high number of these rare cancers and thus to maximise the statistical precision of our study. However, the stratification into different cancer diagnoses and characteristics reduced the statistical precision of the study, and some of the detailed results on individual cancer types have wide confidence intervals.

The mother's educational level was used as a proxy for socioeconomic status, as education remains relatively stable through adulthood and is less affected by changes in health status than income and occupational status (Krieger et al, 1997).

We reduced selection and information bias by using complete registry data to identify all potential childhood cancer patients and their GPs. The distribution of gender, age groups and diagnostic groups in the study population of patients was in concordance with incidence rates formerly reported for childhood cancers (Kaatsch, 2010). The non-response to the questionnaire survey may, however, have introduced selection bias. The mandatory parental approval of the data collection in general practice meant that 173 cases were lost. Analysis of non-responders revealed no statistically significant differences between responders and non-responders apart from the mothers' mean age (data not shown). The parental 
Table 2. The DIs (in days) according to symptoms, clinical intuition, symptom interpretation and wording of the referral letter

\begin{tabular}{|c|c|c|c|c|c|c|}
\hline & \multirow{2}{*}{$\begin{array}{l}\text { Patients } \\
N(\%)\end{array}$} & \multicolumn{2}{|c|}{$\mathrm{DI}$} & \multirow{2}{*}{\begin{tabular}{|c|}
$\begin{array}{c}\text { Nonparametric } \\
\text { test }\end{array}$ \\
P-value
\end{tabular}} & \multicolumn{2}{|c|}{ PR for long DI } \\
\hline & & Median & IQI & & Unadjusted & Adjusted $^{a}$ \\
\hline \multicolumn{7}{|l|}{ Symptoms } \\
\hline \multicolumn{7}{|l|}{ Pain } \\
\hline- & $184(73.0)$ & 17 & $5-47$ & & 1 & 1 \\
\hline+ & $68(27.0)$ & 24 & $3-57$ & 0.862 & $1.1(0.7-1.8)$ & $1.1(0.7-1.8)$ \\
\hline \multicolumn{7}{|l|}{ Fatigue } \\
\hline- & $200(79.4)$ & 20 & $6-61$ & & 1 & 1 \\
\hline+ & $52(20.6)$ & 7 & $2-26$ & 0.003 & $0.4(0.2-0.9)$ & $0.5(0.3-1.1)$ \\
\hline \multicolumn{7}{|l|}{ Recurrent infections } \\
\hline- & $230(91.3)$ & 18 & $4-53$ & & 1 & 1 \\
\hline+ & $22(8.7)$ & 13 & $5-35$ & 0.727 & $0.7(0.3-1.7)$ & $0.9(0.4-2.6)$ \\
\hline \multicolumn{7}{|l|}{ Weight loss } \\
\hline- & $244(96.8)$ & 18 & $5-52$ & & 1 & 1 \\
\hline+ & $8(3.2)$ & 15 & $2-32$ & 0.358 & $0.5(0.1-3.1)$ & $0.6(0.1-3.9)$ \\
\hline \multicolumn{7}{|l|}{ Clinical intuition } \\
\hline- & $221(87.7)$ & 19 & $5-55$ & & 1 & 1 \\
\hline+ & $31(12.3)$ & 10 & $2-26$ & 0.059 & $0.6(0.3-1.4)$ & $0.7(0.3-1.5)$ \\
\hline \multicolumn{7}{|c|}{ Symptom interpretation ${ }^{b}$} \\
\hline Alarm & $48(19.0)$ & 6 & $2-24$ & & 1 & 1 \\
\hline Serious & $126(50.0)$ & 18 & $6-42$ & & $1.8(0.8-4.2)$ & $1.6(0.7-3.5)$ \\
\hline Vague & $64(25.4)$ & 37 & $11-116$ & $<0.001$ & $3.3(1.5-7.3)$ & $3.0(1.4-6.5)$ \\
\hline \multicolumn{7}{|l|}{ Referral wording $^{c}$} \\
\hline Cancer & $64(25.4)$ & 7 & $2-24$ & & 1 & $1.0(0.7-1.4)$ \\
\hline Serious illness & $91(36.1)$ & 16 & $3-62$ & & $2.4(1.1-5.3)$ & $2.2(0.9-4.9)$ \\
\hline 'Something wrong' & 79 (31.3) & 29 & $11-100$ & $<0.001$ & $3.5(1.6-7.4)$ & $3.0(1.4-6.3)$ \\
\hline \multicolumn{7}{|c|}{ 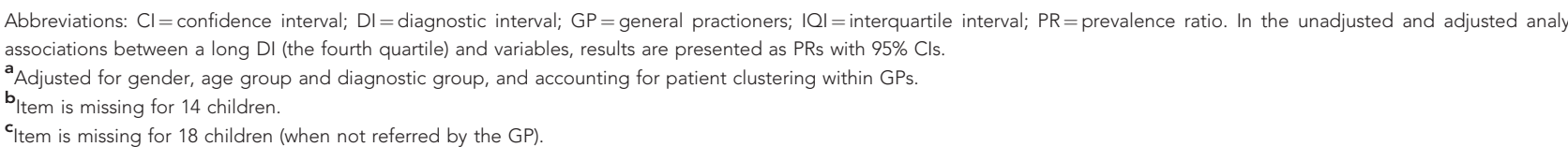 } \\
\hline
\end{tabular}

response rate was satisfactory $(68.5 \%)$, and the GP response rate was high $(86.8 \%)$. Only $3.7 \%$ of the responding parents did not agree to data collection through the Q (GP).

Whenever possible, we used questions from previous surveys (Bjerager, 2006; Hansen et al, 2011), which have already proven effective in describing the Danish population (in terms of milestones and intervals along the diagnostic pathway as well as symptom interpretation); otherwise, new questions were constructed. A high level of content validity of the questionnaires was ensured (Fayers and Machin, 2012) by making maximum use of contacts with GPs, paediatricians and experts within relevant fields and interviewing parents at the Department of Paediatrics, Aarhus University Hospital.

However, the use of retrospective data is a general problem, possibly implying a certain amount of recall bias that may affect both the accuracy of the data (e.g. dates) and the interpretation of the experiences along the cancer trajectory. Accuracy aspects may be particularly relevant in relation to the reported date of symptom onset, the date of first presentation and symptom interpretation. The GPs were encouraged to consult their electronic medical records when completing the questionnaire to reduce such potential information bias. Nearly all Danish GPs have electronic medical records (Protti, 2007). A child may have presented with a chief complaint that was recorded by the GP, whereas less significant symptoms, such as fatigue, may not have been stated in the patient's medical record. Thus, potential 'alarm' symptoms like weight loss and swelling/lump were perhaps more likely to be recorded and reported than vague symptoms. Apart from the item on symptom interpretation and the item on the reason for the encounter-specific symptom, no data on symptom severity were available.

On the other hand, the GP may remember the clinical trajectory rather well because children with cancer are rare in general practice and, in hindsight, they could therefore have added symptoms that were not recorded in the medical record at the time of the encounter.

The study was defined in 2007 and incident childhood cancer patients diagnosed over a 4-year period (2007-2010) were sampled to ensure a sufficient number of study participants. Halfway, in the autumn of 2008, fast-track pathways for children with suspected cancer were implemented in Denmark to decrease delays in diagnosis (National Board of Health Denmark, 2008). GPs' increased focus on the issue due to public awareness of cancer and easier access to advanced diagnostic methods may have influenced the answers provided by GPs. However, subanalyses of our data showed that fast-track pathways were used in $2 \%$ of cases only.

The complete and consecutive inclusion of only patients with a diagnostic pathway involving primary care allows us to consider the results to be generalisable and applicable to other countries 
Table 3. The DIs (in days) according to presenting symptoms and clinical findings for patients with leukaemia, lymphomas or tumours in the CNS ${ }^{a}$

\begin{tabular}{|c|c|c|c|c|c|c|}
\hline & \multirow{2}{*}{$\begin{array}{c}\text { Patients } \\
N(\%)\end{array}$} & \multicolumn{2}{|c|}{ DI } & \multirow{2}{*}{$\begin{array}{c}\begin{array}{c}\text { Nonparametric } \\
\text { test }\end{array} \\
P \text {-value }\end{array}$} & \multicolumn{2}{|c|}{ PR for long DI } \\
\hline & & Median & $|Q|^{a}$ & & Unadjusted & Adjusted $^{\mathrm{b}}$ \\
\hline \multicolumn{7}{|l|}{ Leukaemia } \\
\hline \multicolumn{7}{|l|}{ Symptoms } \\
\hline $\begin{array}{c}\text { Fa tigue } \\
- \\
+ \\
\text { Pain } \\
- \\
+ \\
\text { Recurrent infections } \\
- \\
+ \\
\text { Fever } \\
- \\
+\end{array}$ & $\begin{array}{l}53(63.9) \\
30(36.1) \\
57(68.7) \\
26(31.3) \\
69(83.1) \\
14(16.9) \\
56(67.5) \\
27(32.5)\end{array}$ & $\begin{array}{r}11 \\
6 \\
10 \\
9 \\
10 \\
11 \\
11 \\
7\end{array}$ & $\begin{array}{l}3-27 \\
1-16 \\
2-18 \\
3-45 \\
3-22 \\
2-38 \\
3-27 \\
2-14\end{array}$ & $\begin{array}{l}0.102 \\
0.492\end{array}$ & $\begin{array}{c}1 \\
0.2(0.0-1.7) \\
1 \\
4.4(1.2-16.2) \\
1 \\
2.5(0.7-8.7) \\
1 \\
0.3(0.0-2.0)\end{array}$ & $\begin{array}{c}1 \\
0.2(0.0-1.8) \\
1 \\
5.6(1.6-19.9) \\
1 \\
3.0(0.9-10.3) \\
1 \\
0.2(0.0-1.5)\end{array}$ \\
\hline $\begin{array}{l}\text { Clinical findings } \\
\text { Anaemia } \\
- \\
+ \\
\text { Lymphadenopathy } \\
- \\
+ \\
\text { Bruising } \\
- \\
+ \\
+\end{array}$ & $\begin{array}{l}54(65.1) \\
29(34.9) \\
\\
63(75.9) \\
20(24.1) \\
74(89.2) \\
9(10.8)\end{array}$ & $\begin{array}{r}14 \\
2 \\
11 \\
6 \\
11 \\
2\end{array}$ & $\begin{array}{l}7-30 \\
1-7 \\
3-35 \\
2-23 \\
3-26 \\
1-5\end{array}$ & $<0.001$ & $\begin{array}{c}- \\
- \\
1 \\
0.4(0.1-3.0) \\
- \\
-\end{array}$ & $\begin{array}{c}- \\
- \\
0.4(0.1-2.3) \\
- \\
-\end{array}$ \\
\hline
\end{tabular}

Lymphomas

Symptoms

\begin{tabular}{|c|c|c|c|c|c|c|}
\hline Fatigue & & & & & & \\
\hline - & $32(86.5)$ & 27 & $7-52$ & & - & - \\
\hline+ & $5(13.5)$ & 3 & $2-7$ & 0.039 & - & - \\
\hline Pain & & & & & & \\
\hline $\begin{array}{l}- \\
+\end{array}$ & $29(78.4)$ & 28 & $7-55$ & & - & - \\
\hline $\begin{array}{c}+ \\
\text { Lump/swelling }\end{array}$ & $8(21.6)$ & 6 & $1-17$ & 0.048 & - & - \\
\hline- & $12(32.4)$ & 17 & $10-40$ & & 1 & 1 \\
\hline+ & $25(67.6)$ & 21 & $2-49$ & 0.795 & $1.4(0.3-6.1)$ & $1.8(0.4-7.6)$ \\
\hline $\begin{array}{l}\text { Clinical findings } \\
\text { Anaemia }\end{array}$ & & & & & & \\
\hline - & 34 (91.9) & 23 & $5-49$ & & - & - \\
\hline$\stackrel{+}{+}{ }_{\text {Lymphadenopathy }}$ & $3(8.1)$ & 2 & $0-15$ & 0.100 & - & - \\
\hline $\begin{array}{l}- \\
+\end{array}$ & $27(73.0)$ & $\begin{array}{r}25 \\
5\end{array}$ & $\begin{array}{l}8-55 \\
2-31\end{array}$ & 0100 & 1 & 1 \\
\hline t & $10(21.0)$ & 5 & $2-31$ & 0.199 & & \\
\hline
\end{tabular}

\section{CNS tumours}

\section{Symptoms}

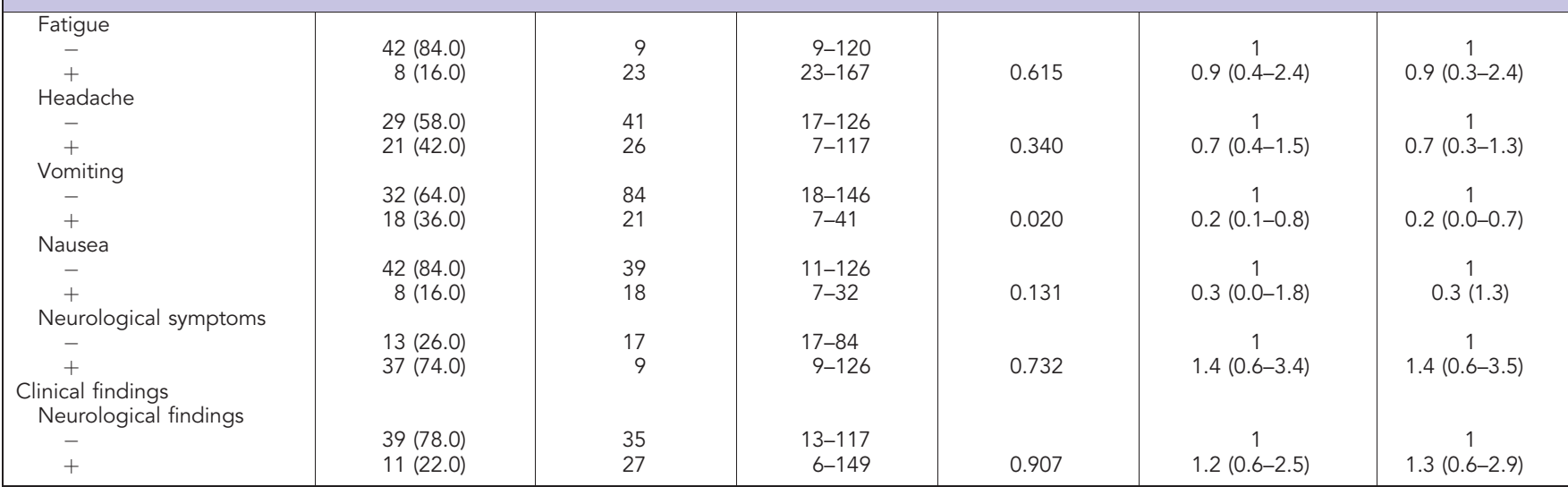

Abbreviations: $\mathrm{Cl}=$ confidence interval; $\mathrm{CNS}=$ central nervous sytem; $\mathrm{DI}=$ diagnostic interval; $\mathrm{GP}=$ general practioner; $|\mathrm{Q}|=$ interquartile interval; $\mathrm{PR}=$ prevalence ratio. When marked by a ' - ', the generalised linear model could not estimate the PR owing to too few cases.

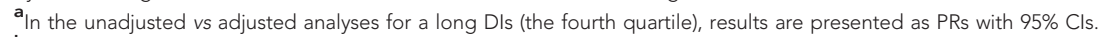

${ }^{\mathbf{b}}$ Adjusted for gender and age group, and accounting for patient clustering within GPs. 
with health-care systems like the Danish, where primary care is the first contact for medical advice and health care.

Comparison with other studies. In the literature, various definitions of time points and intervals along the cancer pathway have been used, and the methods used to describe time intervals are not always clearly explained. A standardised description has only recently been published (Weller et al, 2012). In countries with no gatekeeper system, the time from first symptom presentation (to a health-care professional) to diagnosis is often called the 'physician delay', and this corresponds to the DI in our study. We found a median DI of 18 days (IQI 5-50 days). This is longer than the 8 days (IQI 2-28) reported by a Canadian group (Dang-Tan et al, 2008). We only included children with diagnostic pathways involving a GP. In the remaining cases (62 cases), diagnostic pathways could involve, for instance, emergency cases. Such cases may have had a more rapid progression and more aggressive tumour growth, which would have resulted in statistically significantly shorter DIs (data not shown). A smaller study on children with brain tumours showed a median time interval of 9 days from the first evaluation by a GP to actual referral, that is, this interval was longer than in our study (Klitbo et al, 2011).

In line with our findings, the odds for experiencing a long DI were four times higher in adult cancer patients presenting with vague and uncharacteristic symptoms than in patients presenting with alarm symptoms or any serious symptoms (Torring et al, 2011).

Only few studies have evaluated the association between presenting symptoms and DIs in childhood cancer. A British study showed that longer intervals from symptom onset to diagnosis were associated with head tilt, cranial nerve palsies, endocrine and growth abnormalities, and reduced visual acuity, whereas shorter intervals were associated with nausea and/or vomiting, abnormal gait, coordination difficulties, focal motor weakness and apnoea (Wilne et al, 2012). A German study (Reulecke et al, 2008) on brain tumours revealed that early morning vomiting significantly shortened the time interval from symptom onset to diagnosis, which is in line with our findings. Pain as a symptom may prolong the DI as reported by, for example, Haimi et al (2011). They found time intervals to be longer when the child complained of pain than when the child presented with other symptoms and no pain complaints. Due incorporation of these findings into clinical practice would require more research.

Most authors stress the importance of a timely diagnosis of childhood cancer (Pratt et al, 1978; Dang-Tan et al, 2008; Ansell et al, 2010). However, one study has suggested an inverse relation between symptom duration and the ultimately diagnosed stage of medulloblastoma (Halperin and Friedman, 1996). In other words, the most aggressive tumours tend to have the shortest symptomatic window, a situation that much resembles the 'waiting time paradox' seen in adult cancers (Neal, 2009; Torring et al, 2011). However, documented knowledge of childhood cancers is sparse (DerKinderen et al, 1989). Delay may cause families to lose their trust in the health-care system, and the potential adverse effects of any lost trust on the parent's and the child's well-being and their physical and mental health could easily result in substantial healthcare costs (Dommett et al, 2012).

Conclusion and implications. The GP plays a central role in preventive health care, diagnosis and treatment, and acts as a gatekeeper between the primary and the secondary health-care systems. The GP must assess the risk of critical disease (e.g. cancer) on the basis of several factors, such as presenting symptoms, clinical sense and experience, test results and knowledge of predisposing factors. The GP has also to face diagnostic uncertainty in many situations and yet must fulfil the gatekeeping role in the best possible way.
Appropriate, timely and informative referral from primary to secondary care has become a focus area. This study showed that the GP's symptom interpretation and the wording of the referral letter were highly associated with DI length in cases of childhood cancer. Thus, the GP plays an important role in the timely referral of children with suspected cancer and GPs may help accelerate the diagnostic pathway in secondary care by stating suspicion of cancer or another serious disease.

Owing to national initiatives, fast-track pathways have recently been implemented (National Institute for Health and Clinical Excellence, 2005; National Board of Health Denmark, 2008), and children with suspected cancer must now be seen in secondary health care within $48 \mathrm{~h}$ after referral from general practice. The observed tendency toward a more rapid DI over the study period could perhaps be ascribed to such implementations, but the impact of the initiatives has not yet been fully described.

Diagnosis of childhood cancer is not always straightforward. In primary care, children with cancer may present with symptoms mimicking common and harmless conditions, and the GP continuously faces a diagnostic challenge in identifying the few childhood cancer patients among the many children seen in primary care. This study highlights the need for very detailed medical records, particularly in cases involving children presenting with vague and persistent symptoms.

\section{ACKNOWLEDGEMENTS}

The study was carried out at the Research Unit for General Practice in Aarhus and funded by the Child Cancer Foundation, the Novo Nordisk Foundation, the Danish Cancer Society and the General Practitioners' Foundation for Education and Development. We thank all the participating GPs and parents for their contribution and Ineta Sokolowski for statistical assistance. Questionnaires (in Danish) can be seen at the 'Childhood malignancies - symptoms and DI' project homepage: http://www.cap.au.dk/childhoodcancer

\section{REFERENCES}

Ansell P, Johnston T, Simpson J, Crouch S, Roman E, Picton S (2010) Brain tumor signs and symptoms: analysis of primary health care records from the UKCCS. Pediatrics 125: 112-119.

Barros AJ, Hirakata VN (2003) Alternatives for logistic regression in crosssectional studies: an empirical comparison of models that directly estimate the prevalence ratio. BMC Med Res Methodol 3: 21.

Bjerager M (2006) Delay in diagnosis and treatment of lung cancer. ThesisResearch Unit and Department of General Practice, Faculty of Health Sciences, University of Aarhus: Aarhus, Denmark.

Dang-Tan T, Trottier H, Mery LS, Morrison HI, Barr RD, Greenberg ML, Franco EL (2008) Delays in diagnosis and treatment among children and adolescents with cancer in Canada. Pediatr Blood Cancer 51: 468-474.

DerKinderen DJ, Koten JW, Van Romunde LK, Nagelkerke NJ, Tan KE, Beemer FA, Den OW (1989) Early diagnosis of bilateral retinoblastoma reduces death and blindness. Int J Cancer 44: 35-39.

Dixon-Woods M, Findlay M, Young B, Cox H, Heney D (2001) Parents' accounts of obtaining a diagnosis of childhood cancer. Lancet 357: 670-674.

Dommett RM, Redaniel MT, Stevens MC, Hamilton W, Martin RM (2012) Features of childhood cancer in primary care: a population-based nested case-control study. Br J Cancer 106(5): 982-987.

Fayers PM, Machin D (2012) Score and Measurements: Validity, Reliability, Sensitivity. Wiley: Chichester, UK.

Goyal S, Roscoe J, Ryder WD, Gattamaneni HR, Eden TO (2004) Symptom interval in young people with bone cancer. Eur J Cancer 40: 2280-2286.

Haimi M, Perez-Nahum M, Stein N, Ben Arush MW (2011) The role of the doctor and the medical system in the diagnostic delay in pediatric malignancies. Cancer Epidemiol 35: 83-89. 
Halperin EC, Friedman HS (1996) Is there a correlation between duration of presenting symptoms and stage of medulloblastoma at the time of diagnosis? Cancer 78: 874-880.

Hansen RP, Vedsted P, Sokolowski I, Sondergaard J, Olesen F (2011) Time intervals from first symptom to treatment of cancer: a cohort study of 2,212 newly diagnosed cancer patients. BMC Health Serv Res 11: 284.

Kaatsch P (2010) Epidemiology of childhood cancer. Cancer Treat Rev 36: 277-285.

Klitbo DM, Nielsen R, Illum NO, Wehner PS, Carlsen N (2011) Symptoms and time to diagnosis in children with brain tumours. Dan Med Bull 58: A4285.

Krieger N, Williams DR, Moss NE (1997) Measuring social class in US public health research: concepts, methodologies, and guidelines. Annu Rev Public Health 18: 341-378.

National Board of Health Denmark (2008) Components of Healthcare Relevant to Urgent Referral Pathways for Children with Suspected Cancer. National Board of Health Denmark: Denmark.

National Institute for Health and Clinical Excellence (2005) Referral Guidelines for Sucpected Cancer in Adults and Children. National Institute for Health and Clinical Excellence.

Neal RD (2009) Do diagnostic delays in cancer matter? Br J Cancer 101(Suppl 2): S9-S12.

Olivarius NF, Hollnagel H, Krasnik A, Pedersen PA, Thorsen H (1997) The Danish National Health Service Register. A tool for primary health care research. Dan Med Bull 44: 449-453.

Pedersen CB, Gotzsche H, Moller JO, Mortensen PB (2006) The Danish Civil Registration System. A cohort of eight million persons. Dan Med Bull 53: 441-449.

Pratt CB, Smith JW, Woerner S, Mauer AM, Hustu HO, Johnson WW, Shanks EC (1978) Factors leading to delay in the diagnosis and affecting survival of children with head and neck rhabdomyosarcoma. Pediatrics 61: $30-34$.

Protti D (2007) Comparison of information technology in general practice in 10 countries. Healthc Q 10: 107-116.

Punt J (2004) Clinical syndromes. In Brain and Spinal Tumours of Childhood, Walker D, Perilong G, Punt J et al. (eds) pp 99-106. Arnold: London, UK.

Raab CP, Gartner Jr JC (2009) Diagnosis of childhood cancer. Prim Care 36: 671-684.

Reulecke BC, Erker CG, Fiedler BJ, Niederstadt TU, Kurlemann G (2008) Brain tumors in children: initial symptoms and their influence on the time span between symptom onset and diagnosis. J Child Neurol 23: 178-183.

Torring ML, Frydenberg M, Hansen RP, Olesen F, Hamilton W, Vedsted P (2011) Time to diagnosis and mortality in colorectal cancer: a cohort study in primary care. Br J Cancer 104(6): 934-940.

Weller D, Vedsted P, Rubin G, Walter FM, Emery J, Scott S, Campbell C, Andersen RS, Hamilton W, Olesen F, Rose P, Nafees S, van RE, Hiom S, Muth C, Beyer M, Neal RD (2012) The Aarhus statement: improving design and reporting of studies on early cancer diagnosis. $\mathrm{Br} J$ Cancer 106(7): 1262-1267.

Wilne S, Collier J, Kennedy C, Jenkins A, Grout J, Mackie S, Koller K, Grundy R, Walker D (2012) Progression from first symptom to diagnosis in childhood brain tumours. Eur J Pediatr 171: 87-93.

World Health Organization (WHO) (2011) International Classification of Diseases (ICD). Available at: http://www.who.int/classifications/icd/en/.

This work is published under the standard license to publish agreement. After 12 months the work will become freely available and the license terms will switch to a Creative Commons AttributionNonCommercial-Share Alike 3.0 Unported License. 